INPLASY

PROTOCOL

To cite: Zhang et al. Effect of Znic Supplementation on Patients with Chronic Heart Failure: A systematic Review and Meta Analysis. Inplasy protocol 202210100. doi: 10.37766/inplasy2022.1.0100

Received: 19 January 2022

Published: 19 January 2022

Corresponding author: Ming-JIn Zhu

zhumingjin@zcmu.edu.cn

Author Affiliation:

Tongde Hospital of Zhejiang Province.

Support: No.

Review Stage at time of this submission: Preliminary searches.

Conflicts of interest:

None declared.

\section{Effect of Znic Supplementation on Patients with Chronic Heart Failure: A systematic Review and Meta Analysis}

Zhang, B1; Zhang, M2; Lu, CJ3; Liu, LZ4; Jiang, C5; Zhu, MJ6.

Review question / Objective: To investigate whether znic supplementation is associated with lower mortality in patients with chronic heart failure.

Information sources: Databases: Medline (Ovid), Embase (Ovid), the Cochrane Central Register of Controlled Trials (CENTRAL), ClinicalTrials.gov and the World Health Organization International Clinical Trials Registry Platform to identify ongoing or unpublished eligibletrials.

INPLASY registration number: This protocol was registered with the International Platform of Registered Systematic Review and Meta-Analysis Protocols (INPLASY) on 19 January 2022 and was last updated on 19 January 2022 (registration number INPLASY202210100).

\section{INTRODUCTION}

Review question / Objective: To investigate whether znic supplementation is associated with lower mortality in patients with chronic heart failure.

Condition being studied: Randomised controlled trials comparing Znic supplementation with a placebo or no treatment for mortality were included. Independent data extraction was conducted and study quality assessed. A meta analysis was carried out by using fixed effects and random effects models to calculate risk ratio of death in the group receiving znic supplementation and the control group.

\section{METHODS}

Search strategy: One of the authors conducted the search of several databases: Medline (Ovid), Embase (Ovid), 
the Cochrane Central Register of Controlled Trials (CENTRAL), from inception to 31 December 2021. We also searched ClinicalTrials.gov and the World Health Organization International Clinical Trials Registry Platform to identify ongoing or unpublished eligible trials. To maximise the search for relevant articles, we reviewed reference lists of identified trials and systematic reviews. We did not apply language restrictions.

Participant or population: Patients with chronic heart failure.

Intervention: Znic supplementation

Comparator: Chronic heart failure patients with a placebo or no treatment

Study designs to be included: Randomised controlled trials comparing Znic supplementation with a placebo or no treatment.

Eligibility criteria: Randomised controlled trials comparing vitamin Dsupplementation with a placebo or no treatment formortality were included. Independent data extraction was conducted and study quality assessed.

Information sources: Databases: Medline (Ovid), Embase (Ovid), the Cochrane Central Register of Controlled Trials (CENTRAL), ClinicalTrials.gov and the World Health Organization International Clinical Trials Registry Platform to identify ongoing or unpublished eligible trials.

\section{Main outcome(s): All cause mortality.}

Quality assessment / Risk of bias analysis: Two researchers independently assessed the quality of all included trials by using the Cochrane Collaboration risk of bias tool. They also examined the quality of evidence for outcomes using the grading of recommendations assessment, development, and evaluation (GRADE) approach.

Strategy of data synthesis: We performed statistical analyses using RevMan (version 5.3.3; The Cochrane Collaboration) and the meta package in $\mathbf{R}$ (version 3.4.3; R Project for Statistical Computing). Analyses for all outcomes were conducted on an intention to treat basis. We used risk ratios and their associated $95 \%$ confidence intervals to assess outcomes, and considered a P value less than 0.05 to be statistically significant. We assessed heterogeneity using the 12 test. If significant heterogeneity was not present $(12<50 \%)$, we used fixed effects models to pool outcomes; we used random effects models when significant heterogeneity was present $(12 \geq 50 \%)$. The possibility of small study effects was assessed qualitatively by visual estimate of the funnel plot and quantitatively by calculation of the Egger test, the Begg test, and the Harbord test.

Subgroup analysis: We performed several subgroup analyses to test interactions according to dose ; type of znic; timing of treatment (daily and intermittently); and mean age ( $\geq 70$ and $<70$ years). We conducted retrospective subgroup analyses based on length of follow-up (at least three years and less than three years); year of publication (before 2015 and in or after 2015); sex (female and both sexes); residential status (community and institution); bolus (yes and no).

Sensitivity analysis: We conducted sensitivity analyses by excluding trials with high or unknown risk of bias; excluding trials with high risk or unknown risk of bias of the different domains; excluding quasi randomised or cluster randomised trials; excluding the largest trial; excluding trials with a follow-up of less than one year; using random effect models.

\section{Country(ies) involved: China.}

Keywords: Zn, Znic, Znic supplementation, Chronic heart failure, Systematic review, Meta analysis

Contributions of each author:

Author 1 - Bin Zhang - Conceived the study and designed the protocol and selected the studies and extracted the relevant information.

Email: 452551332@qq.com 
Author 2 - Ming Zhang - Conceived the study and designed the protocol and selected the studies and extracted the relevant information.

Email: zhangming8298@126.com

Author 3 - Cao-Jie Lu - Performed the literature search and synthesised the data and wrote the first draft of the paper.

Email: 450122361@qq.com

Author 4 - Li-Zhi Liu - Selected the studies and extracted the relevant information and synthesised the data and wrote the first draft of the paper.

Email: 273782406@qq.com

Author 5 - Chen Jiang - Selected the studies and extracted the relevant information and synthesised the data and wrote the first draft of the paper.

Email: jiangchen666@163.com

Author 6 - Ming-Jin Zhu - conceived the study and designed the protocol.

Email: zhumingjin@zcmu.edu.cn 